\title{
The Influence of the Acoustic Reflector Design on the Temperature Coefficient of Frequency for Shear and Longitudinal Mode AIN Resonators
}

\author{
Junaid Munir, Mario DeMiguel-Ramos, Hyunjoo Jenny Lee, Mohammad Alam Saeed, and Enrique Iborra
}

\begin{abstract}
Most of the applications of solidly mounted resonator need the compensation of temperature coefficient of resonant frequency. The presence of materials with different properties in the stack makes it complicated to explain the influence of each material individually on temperature coefficient. To reduce design and fabrication costs, an accurate and precise modeling of the resonators is required. In this paper, Mason model and finite-element analysis are used to design the resonators and results for both models, which are almost similar, are presented. Different kinds of configuration (symmetric and asymmetric, according to the thickness of the layers) are designed to achieve the temperature coefficient of frequency as near-zero as possible with an optimized response. Such designs are fabricated and characterized obtaining measurements of the temperature coefficient of frequency. The result shows that useful and reliable information on the performance of resonators can be achieved with accurate modeling.
\end{abstract}

Index Terms-Solidly mounted resonators (SMRs), temperature coefficient of frequency (TCF), mason model, finite element modeling (FEM).

\section{INTRODUCTION}

B ULK ACOUSTIC wave resonator (BAW) based filters have gained substantial interest for many RF applications. They have decisive advantages and superior performance in chip size and processing costs as compared to surface acoustic wave (SAW) and ceramic filters [1]. With the growth of wireless communication technology, specification compliance over a wide range of temperature is an essential current necessity. One of the most limiting parameters in filter technology is the temperature variations of clue frequencies, which are fixed by the resonant and anti-resonant frequencies of resonators. Additionally, BAW resonators are also emerging as very sensitive gravimetric sensors for chemical and biochemical species [2], [3]. In this application, the variations of the resonant frequency (where the output of the sensor lies) with temperature is especially dramatic as the expected sensitivities are in the range of very few ppm variations of the resonant frequency and can be masked by them. For in liquid operation, that is the most common situation in biosensors, resonators need to operate in shear acoustic modes [4]. Therefore, it is very interesting to study not only the behavior of the common longitudinal acoustic modes of a BAW resonators used in RF-electronics and gas sensing, but also the shear mode one used for in-liquid biochemical sensing. In recent studies, bulk acoustic resonator based sensors also show promising applications in blood coagulation monitoring technology [5], [6].

The thermal stability of the resonator can be assessed by the temperature coefficient of the resonant frequency (TCF) of the working mode. It indicates the relative change of resonant frequency with change in temperature $(\mathrm{TCF}=(1 / f)$. $(\mathrm{d} f / \mathrm{d} T)$ ) [7]. In [8], the degradation of TCF in a band $7 \mathrm{Tx}$ filter application is investigated. Between the different types of BAW resonators, solidly mounted resonators (SMR), which uses an acoustic reflector for acoustically isolating the device from the substrate, are the preferred option for most of the industrial producers of filters and duplexers [9]. TCF depends on the temperature coefficients of the material properties that fixes the frequency of resonance of the device such as the elastic constants, linear dilatation coefficients, and mass density of all the materials involved [10]. The elastic constants, which directly fix the acoustic velocity, are the most influent properties because they change much more than the other properties with temperature. In a complex layered structure such as a SMR, all the layers influence the TCF in more or less extent, depending on the amount of acoustic energy entering into them. The piezoelectric layer is the most influent, followed by the electrodes. The electrode's influence on the performance of the resonator is studied in shear mode with high Q factor [11]. Between the reflector layers the influence on the TCF is more important as the layer is nearer the piezoelectric one because the acoustic energy that arrive to each layer is a fraction of that arriving to the upper one due to the reflection of waves in the interface. To reduce TCF and avoid malfunction of the device, one has to design the layout by using materials that compensate the variations of the frequency with temperature of the other ones. For instance, in a BAW resonator, which 
use AlN as piezoelectric, the TCF is negative, however if a thin film of $\mathrm{SiO}_{2}$ is deposited on the top of the resonator, the TCF increases towards less negative values because the temperature coefficient of the acoustic velocity in $\mathrm{SiO}_{2}$ is positive. However, this solution does not always work as desired because other properties of the resonator such as the effective electromechanical coupling factor $\left(k_{\text {eff }}^{2}\right)$ and the quality factor $(\mathrm{Q})$ strongly reduce making the device performances to be worse. In the literature there are many examples of how to compensate the TCF of BAW resonators. A direct bonding technique is used for the compensation of TCF in SAW which provides that a thin layer of piezoelectric material can leads to a smaller TCF [12]. In [13], the $\mathrm{SiO}_{2}$ layer is deposited on the bottom electrode under the piezoelectric film. Part of the applied electric field drops in the $\mathrm{SiO}_{2}$ layer, dramatically reducing the $k_{\text {eff }}^{2}$ from $6 \%$ to $1.5 \%$. If the layer is deposited on the top electrode as in [14], the reduction of TCF is obtained with thicker $\mathrm{SiO}_{2}$ layers but the reduction in $k_{\text {eff }}^{2}$ is lower. In [15], a complex hybrid structure based on an acoustic reflector and an air cavity is designed to reduce the negative $\mathrm{TCF}$ from $-25 \mathrm{ppm}{ }^{\circ} \mathrm{C}$ to $-11 \mathrm{ppm} /{ }^{\circ} \mathrm{C}$ without an important $k_{\text {eff }}^{2}$ degradation. Fluorine-doped silicon oxide (SiOF) is used between piezoelectric layers with different configurations and $k_{\text {eff }}^{2}$ up to $6.26 \%$ is achieved with $\mathrm{TCF}$ of $-11 \mathrm{ppm} /{ }^{\circ} \mathrm{C}$ in temperature compensated FBARs [16].

In the above described literature, different methods were used for the compensation of TCF but the effect of the acoustic reflector layers on the TCF and on overall performance of the resonator is limited. In our current approach, we compensate the TCF with just carefully adjusting the thickness of first two acoustic reflector layers without using an additional $\mathrm{SiO}_{2}$ layer which is not done before.

In the particular case of SMR, because the structure contains more layers and materials than in a suspended resonator, the possibilities of tailoring the thermal behavior and maintaining high enough performances increase without making more complex the fabrication technology.

In this paper, we study by numerical simulations and by experimental measurements the influence of the different layers of the acoustic reflector on the TCF of an SMR. We have designed and fabricated different acoustic reflectors with the thickness of the layers different from the ideal quarterwavelength (the so called Bragg reflectors) and fabricated on them AlN resonators with the aim of reducing the absolute value of the TCF but maintaining the resonator performances as high as possible. Longitudinal and shear modes of operation have been investigated by using $\mathrm{c}$-axis oriented or c-axis tilted AlN films respectively. In parallel, we have simulated the SMR response by using the Mason's model with only the acoustic velocity of the materials being dependent on the temperature. Also some simulations by finite elements modelling (FEM) using COMSOL software with all the parameters being dependent with temperature have been made to confirm the accuracy of the Mason's model simulations. Two kind of acoustic reflectors are studied. Both use $\mathrm{SiO}_{2}$ as low acoustic impedance material. The first uses Mo as high acoustic impedance material and the second uses electrically insulating amorphous $\mathrm{Ta}_{2} \mathrm{O}_{5}$ as high impedance material. Anomalous

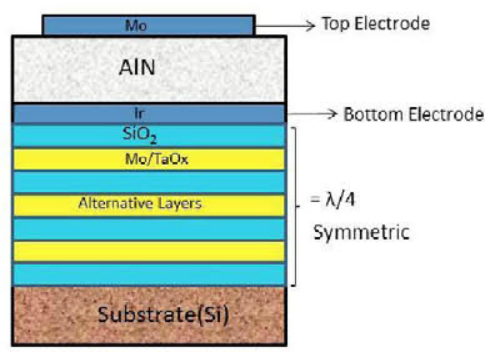

(a)

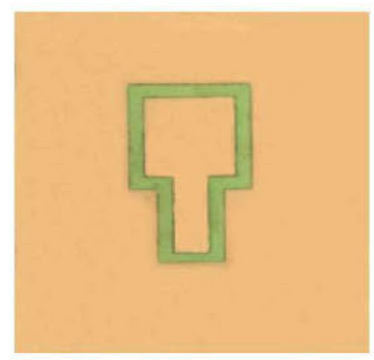

(b)
Fig. 1. (a) Cross section representation of the SMR device, (b) optical microscopic image of the top view.

behavior in the second type of resonators has been found. The cross section representation for the symmetric configuration in the case of $\mathrm{SiO}_{2} / \mathrm{Mo}$ based resonator and the microscopic image of the fabricated devices is shown in Fig.1.

\section{Simulation Methods}

We have used in this work the one-dimensional Mason's physical model to obtain the TCF by simulating the response of the resonators changing the values of the material properties with temperature. For comparison purposes, we have also used a more complex FEM multi-physics application i.e. COMSOL, which uses the whole effects occurring and is able to simulate 2D and 3D systems. We have verified that the TCF results obtained by both simulation methods are almost identical in some representative cases. Therefore, most of the simulations were made with the Mason's model because it is much less time-consuming.

One-dimensional Mason's model [17] offers a simple way of determining the main characteristics of the resonators (resonant frequencies, electromechanical coupling factors, and quality factors). It is possible to operate in both shear and longitudinal modes by assuming the same capacitive coupling for them. Each mode is defined by the different material properties (acoustic velocity, mass density and acoustic losses). According to this model, the acoustic behavior of the reflector in a SMR can be modeled by considering at each frequency that there are waves traveling in each direction. The propagating velocity of these waves are characteristic of each material forming the stack and in each interface between two different materials, there is a reflection, which is given by

$$
R=\frac{Z_{u}-Z_{d}}{Z_{u}+Z_{d}}
$$

Where $Z_{u}$ is the acoustic impedance of all the stack over the considered interface and $Z_{\mathrm{d}}$ is the acoustic impedance of all the stack under it. All $\mathrm{R}, \mathrm{Z}_{\mathrm{u}}$, and $\mathrm{Z}_{\mathrm{d}}$ are complex numbers resulting from the product of mass density and acoustic velocity, including mechanical losses. By applying this method, the acoustic response of the whole structure can be calculated. In [18], the optimization of acoustic mirrors in SMRs not only reduces the insertion loss but also improve Q-factor.

The acoustic behavior of each layer can be simulated by an electrical model shown in Fig.2(a) where the input 


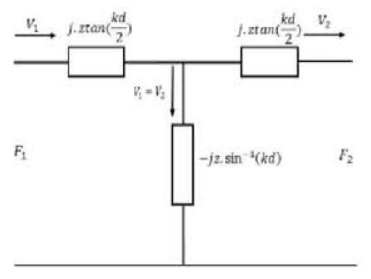

(a)

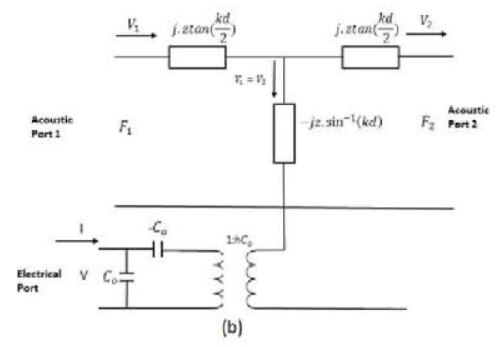

Fig. 2. Circuital expresions of the Mason's model for a) a non piezoelctric layer and b) a piezoelctrica layer, showing the elements which simulate the acosutic behaviour (in both cases) and those which simulate the electrical behaviour in the piezoelctric layer.

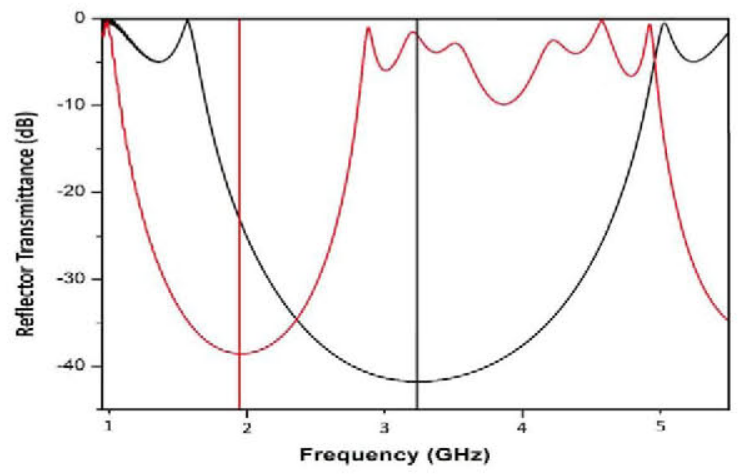

Fig. 3. Modulus of the transmittance spectrum of a $3.3 \mathrm{GHz}$ centered 2/4-reflector made with two pair $\mathrm{Mo}-\mathrm{SiO}_{2}$ alternate layers, (black line for longitudinal and red line for shear waves). The vertical lines indicate the center of the transmission bands.

and output parameters are forces per unit area and strains. In the case of the piezoelectric layer, shown in Fig.2(b), an electrical port is added to take into account the piezoelectric effect.

The modulus of the reflector reflectance can be directly drawn as a function of frequency [19]. However, we depict the modulus of the reflector transmittance (in $\mathrm{dB}$ ), as given by

$$
|T|=10 \cdot \log (1-|R|)
$$

In this way, the reflector response can be better analyzed. The calculated reflector transmittance as a function of frequency of a $\lambda / 4, \mathrm{Mo}-\mathrm{SiO}_{2}$ reflector is shown in the Fig.3. The needed material constants were previously measured [20]-[22].

The piezoelectric capacitor is located on top of this reflector. In the piezoelectric layer, the deformations generated by the acoustic waves traveling inside it causes, by the direct piezoelectric effect, an additional electric field, which has the consequence of varying the ratio between the total electric field and the flowing current, that is a variation of the electrical impedance. This is well modeled by the Mason's model, which considers the piezoelectric layer as a block with two acoustic ports and one electrical port, which takes into account the piezoelectric effect.

First, we have verified that 1D Mason's model and 2D FEM give the same simulated results for a resonator at room temperature. Fig. 4 shows the impedance spectra around the resonant frequency for shear and longitudinal modes of a
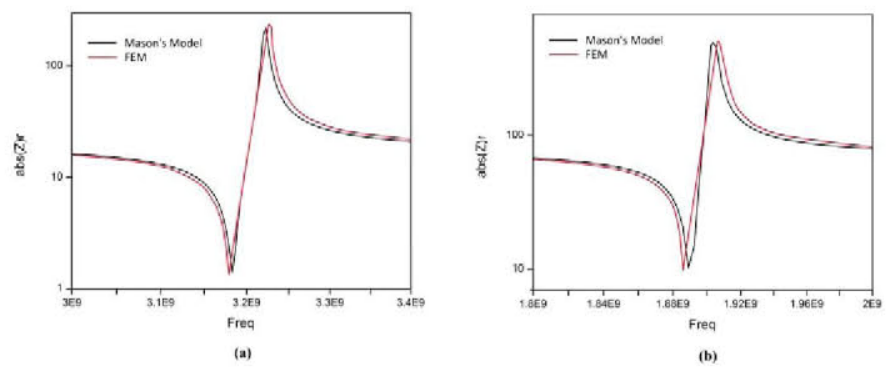

Fig. 4. Simulated results of the impedance as a function of frequency for longitudinal (a) and shear (b) wave modes.

device designed by using a $\mathrm{SiO}_{2} / \mathrm{Mo}$ based reflectors. The analyzed thicknesses of the top and bottom electrodes are $0.15 \mu \mathrm{m}$ and $0.14 \mu \mathrm{m}$ respectively. The thickness of $\mathrm{AlN}$ is $0.8 \mu \mathrm{m} . \mathrm{SiO}_{2}$ and Mo have $0.4697 \mu \mathrm{m}$ and $0.4765 \mu \mathrm{m}$ thick layers for the symmetric configuration, respectively. The minimum of the impedance modules is reached at the resonant or series frequency $\left(f_{\mathrm{s}}\right)$ and the maximum at the anti-resonant or parallel frequency $\left(f_{\mathrm{p}}\right)$. The displacement amplitude, which is constant inside the resonator and exponentially decreases outside it, does not meet the continuity conditions at the resonator edges. This causes to excite the various spurious modes having non vanishing lateral propagation. To avoid it, an additional border region is usually introduced [23]. It decreases the content of spurious modes because of its lower Eigen-resonance frequency.

Additionally, some simulations using FEM without any initial hypothesis (i.e. all the properties having a temperature dependence) was done to compare results with Mason's model simulations taking into account only the thermal variation of the sound velocity. On the other hand, the shape and finite lateral size of the top electrode may cause the deterioration of overall performance of the resonator because surface acoustic waves can travel parallel to the surface and reflect on the top electrode edges generating additional resonances, which also would be seen in the impedance spectrum. This can only be taken into account by precise 2D or 3D numerical modeling of the resonators. The behavior of a physical system for external loads can be predicted with computer-aided numerical method, i.e. FEM. In our model, COMSOL solves the multi-physics problem including electrical and mechanical equations in the piezoelectric layer. The electrical equations are not solved in the metallic Mo layer because the electrical conductivity of these layers is higher than that of AlN. Hence, the piezoelectric layer only contains the dominant electromechanical coupling. The idea is that to observe the effects of different materials and their thicknesses in the stack on the TCF of the resonators. The thicknesses of the piezoelectric layer and the electrodes have been also varied in order to get the maximum response from the resonators.

In this work, both shear and longitudinal wave modes are simulated and measured with variable thicknesses of different layers to study the evolution of the respective TCF. As we mentioned above, the influence of the temperature variations on the resonant frequency is due to the variations of all the properties of all the material which compose the resonator, 
from the substrate to the top electrode. However, not all the material properties have the same influence on the thermal variation of the resonant frequency and not all the parts of the layered structure forming the SMR have the same weight in this influence. Piezoelectric constants, dielectric constants (including electrostriction), linear dilatation coefficients and elastic constants (shear and bulk elastic modulus) are the properties that influence the resonant frequency of an SMR. In a first approach, for a single piezoelectric layer without electrodes, the resonant frequency is fixed by the thickness and the acoustic mode propagating velocity. Therefore, the acoustic velocity (proportional to the squared root of the ratio of the elastic modulus and the mass density) and the thickness are the only properties in this simple case. When more and more layers are added to this piezoelectric layer, the resonant frequency varies due to mass loading effect and also more factors influence its thermal behavior. A first evaluation of the influence of each of the mentioned properties proves that variation of the elastic constants (sound velocity) is by far the most important one. In fact, the sum of all the thermal effects of all properties except the variations of the elastic constants (acoustic velocities) is at least two orders of magnitude lower than those of the acoustic velocity alone. Therefore, to simplify the models for saving computation time we have only considered in the Mason's model, the acoustic velocity variations with temperature of all the layers of the SMR.

Acoustic waves generated in the piezoelectric layer travel in both directions suffering reflections in each layer interface according to equation (1). As interferences occur, there are some frequencies at which a standing wave is generated in each layer, giving a maximum in the acoustic signal. In the piezoelectric layer, this effect is the origin of the main resonance which is commonly measured by monitoring the variations of the electrical impedance with frequency. In the other layers, which are not piezoelectric, there is not added internal electrical field and their electrical impedance does not change. However, if the perturbation at the frequency where the standing wave occurs is strong enough, the piezoelectric layer, acting as a microphone, give an electrical signal at that frequency. These additional resonances, occurring in each layer, are detected in the piezoelectric one and they are the so called overtones. To an overtone be visible in the device electrical impedance spectrum, it should deform the piezoelectric layer with enough intensity. This occurs if the overtone frequency lies inside the acoustic reflector band. Additionally, the intensity of the overtone depends on the amount of acoustic energy supply to the particular layer. Therefore, if the overtone is generated in a deep layer, far from piezoelectric capacitor, the amount of acoustic energy is very low making the electrical impedance variation in the piezoelectric layer very small. It is worth to note that any acoustic wave traveling through the device, partially reflects in each material interface, being the transmitted amplitude lower each time. Fig.5 show this effect using finite element modeling. It also shows the total energy including the strain and electrical energy.

In most of our samples, with one of the two uppermost layers with thickness higher than the corresponding

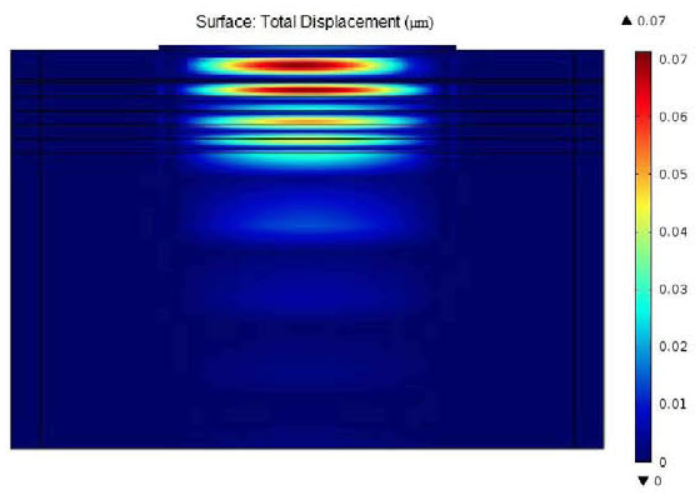

Fig. 5. Displacement profile of the total acoustic energy distribution in the SMR.

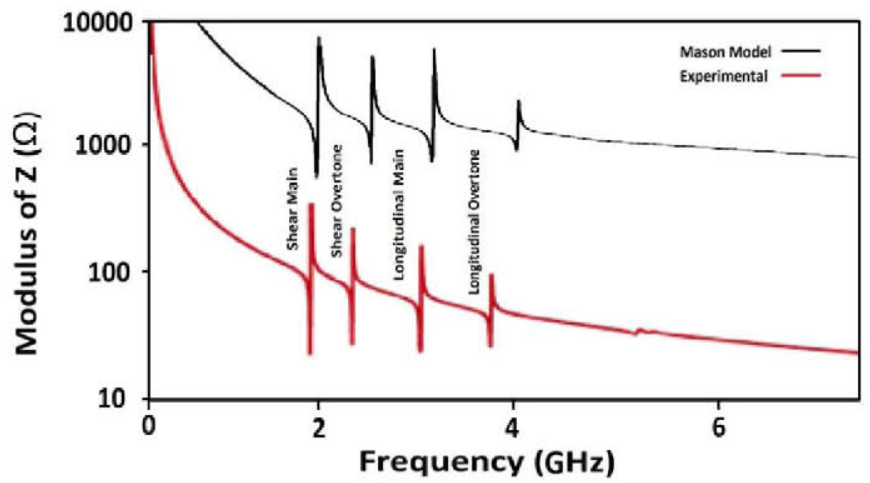

Fig. 6. Simulated and Measured spectrum of the electrical impedance modulus of a SMR made with tilted grain AlN film and an acosutic reflector with uppermost $\mathrm{SiO}_{2}$ layer of more than twice quarter wavelength thickness. Model results are verticaly displaced for clarity.

quarter-wavelength which is for each layer $v_{\mathrm{a}} /\left(4 \cdot f_{\mathrm{c}}\right)$ being $v_{\mathrm{a}}$ the acoustic velocity of the considered material and $f_{\mathrm{c}}$ the center frequency of the reflector, the resonant frequency of the overtone for each mode appears in the electrical impedance spectrum. Fig. 6 shows the simulated and measured electrical impedance spectrum of a sample, which illustrate this behavior. This resonator was made with tilted grains AlN (shear and longitudinal modes are excited) and it has the uppermost low acoustic impedance layer of $\mathrm{SiO}_{2}$ of a thickness more than the double of the corresponding quarter-wavelength. The spectrum shows the main resonances in AlN film (shear and longitudinal) and the overtones in the uppermost reflector layer (shear and longitudinal).

The simulated and measured thickness of piezoelectric layer, top and bottom electrode and first acoustic reflector layer for electrical impedance spectrum (Fig.6) are given in the table I. The remaining reflectors thickness are corresponding to the $\lambda / 4$ of the respective frequency (i.e. $3.3 \mathrm{GHz}$ ).

\section{EXPERIMENTAL}

SMRs have been fabricated by depositing AlN piezoelectric capacitors on different acoustic reflectors made of alternating layers of low and high acoustic impedance materials. An ultrahigh-vacuum system was used to sputter AlN films. The base pressure was always below $1.3 \times 10^{-6} \mathrm{~Pa}$. A high purity 
TABLE I

Simulated AND MEAsured THickness of THE MATERIALS FOR THE ELECTRICAL IMPEDANCE SPECTRUM

\begin{tabular}{lcc}
\hline \hline \multicolumn{1}{c}{ Materials } & $\begin{array}{c}\text { Simulated Thickness } \\
(\mu \mathrm{m})\end{array}$ & $\begin{array}{c}\text { Measured Thickness } \\
(\mu \mathrm{m})\end{array}$ \\
\hline Top Electrode & 0.15 & - \\
AIN & 0.8 & 0.75 \\
Bottom Electrode & 0.14 & - \\
$1^{\text {st }}$ acoustic reflector & 1.21 & - \\
$\left(\mathrm{SiO}_{2}\right)$ layer & &
\end{tabular}
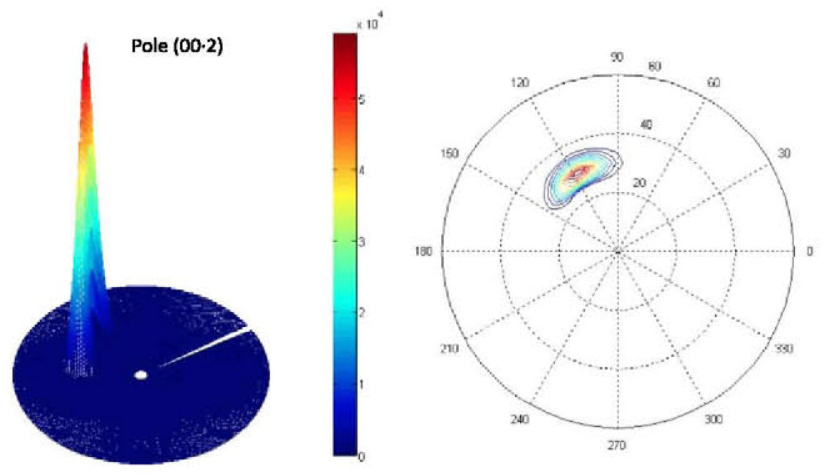

Fig. 7. X-ray diffraction pole figure of the $(00 \cdot 2)$ reflection of a typical tilted grains AlN film.

aluminum target $150 \mathrm{~mm}$ in diameter was sputtered in $\mathrm{Ar} / \mathrm{N}_{2}$ atmospheres (40:60) using a pulsed-DC power source (MKS ENI RPG-50E) operating at $250 \mathrm{kHz}$. Pure c-axis oriented and tilted c-axis AIN films were used for study longitudinal and shear modes. Pure c-axis oriented films were sputtered at $1.2 \mathrm{~kW}$ and low pressure $(0.27 \mathrm{~Pa})$; the substrate was kept at $400{ }^{\circ} \mathrm{C}$ during deposition and for biasing the substrate to a DC voltage of $-60 \mathrm{~V}$, a RF power of $15 \mathrm{~W}$ was used to adjust the in plane residual stress to values lower than $200 \mathrm{MPa}$. By applying these conditions, films grew in the center of the substrate at a rate of $45 \mathrm{~nm} / \mathrm{min}$. To deposit AlN films with tilted grains, before the main AIN film deposited in the same conditions, an AlN seed layer was deposited at high pressure $(0.66 \mathrm{~Pa})$ and low power of $600 \mathrm{~W}$ without deliberately biasing or heating the substrate. Seed layers of AlN exhibit (1.03) preferred orientation, which was crucial to stimulate the growth of the consequent AIN films with tilted grains respect the normal to the surface provided by these conditions [24]. Additionally, the samples were located at $5 \mathrm{~cm}$ from the center of the substrate holder to obtain directional deposition and only $34 \mathrm{~nm} / \mathrm{min}$ of deposition rate was achieved. This seed layer promoted the growth of AIN films with the wurtzite $\mathrm{c}$ axis tilted up to $30^{\circ}$ with respect to the surface normal in the substrates placed between $2 \mathrm{~cm}$ and $5 \mathrm{~cm}$ from the target axis. In Fig.7 the x-ray diffraction pole figure of the $(00 \cdot 2)$ reflection of a typical tilted grains AlN film is shown, showing a tilt angle of the c-axis of around $30^{\circ}$.

As electrodes we use Ir (140 nm) as bottom and Mo $(150 \mathrm{~nm})$ as top. Bottom electrode is continuous and at the

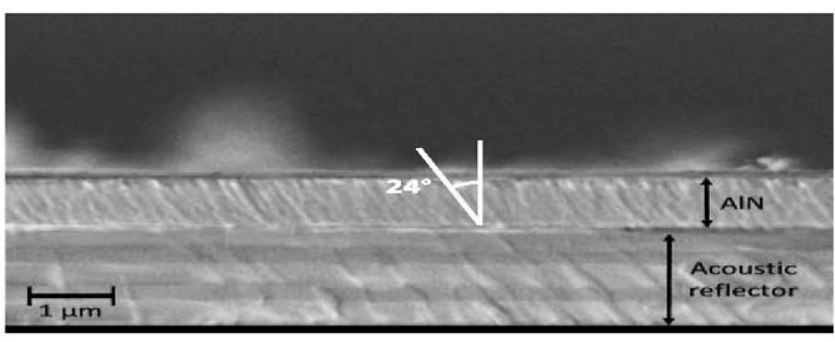

Fig. 8. SEM cross section image of a typical resonator.

same plane than the top electrode an extended ground plane capacitively couples it. The top electrode has been patterned by dry-etching.

(100) oriented silicon wafers were used as substrate. After cleaning the acoustic reflector was deposited by sputtering alternately, in the same vacuum cycle, layers of the different material tested. $\mathrm{SiO}_{2}$ was always use as low impedance material. It was deposited from a Si target in $\mathrm{Ar}: \mathrm{O}_{2}$ mixtures at high oxygen content in the discharge $(\sim 70 \%)$ and low pressure to ensure a dense material with positive temperature coefficient of the acoustic velocity. As high acoustic impedance material $\mathrm{Mo}$ and amorphous $\mathrm{Ta}_{2} \mathrm{O}_{5}$ were used. The total number of layers was always seven. The thickness of each layer was previously calculated using Mason's model. For the design of the one port test devices we chose a resonant frequency for the longitudinal mode of the symmetric reflector resonator of $3.3 \mathrm{GHz}$ (half for the shear mode), which is achieved with an AlN thickness of $750 \mathrm{~nm}$. The area of the devices was $16000 \mu \mathrm{m}^{2}$. The electromechanical coupling factor for shear and longitudinal mode depend on the structure of the acoustic resonator, for the symmetric one, they are around $2.5 \%$ and $3.5 \%$ respectively. In Fig. 8 a SEM cross section image is shown.

The electrical impedance of the SMRs was measured as a function of the frequency on-wafer with $150 \mu \mathrm{m}$ pitch RF-probes Picoprobe from GGB Industries with an Agilent N5230A network analyzer using a driving power of $-5 \mathrm{dBm}$. We use segmented frequency scans to acquire 4000 samples around each resonance for accurately determining the resonant frequency. The sample temperature was varied during measurements from $25^{\circ} \mathrm{C}$ to $100^{\circ} \mathrm{C}$ in steps of around $10^{\circ} \mathrm{C}$ by a heater in the sample holder and a temperature controller. A type-K thermocouple was used to measure the temperature of the sample by attaching it with a high thermal conductive paste to its surface.

\section{RESUlts AND Discussion}

In the present work, a theoretical as well as experimental study of the compensation of TCF using different materials in acoustic reflectors with 1D Mason model and 2D (FEM) is presented. We start with a symmetric $\lambda / 4$-mode reflector configuration [25], the so called Bragg reflector, in which each layer is quarter acoustic wavelength thick for the central frequency, i.e. the thickness of each layer is layer $v_{\mathrm{a}} /\left(4 \cdot f_{\mathrm{c}}\right)$ being $v_{\mathrm{a}}$ the acoustic velocity of the considered material and $f_{c}$ the center frequency of the reflector. Then, we study the 


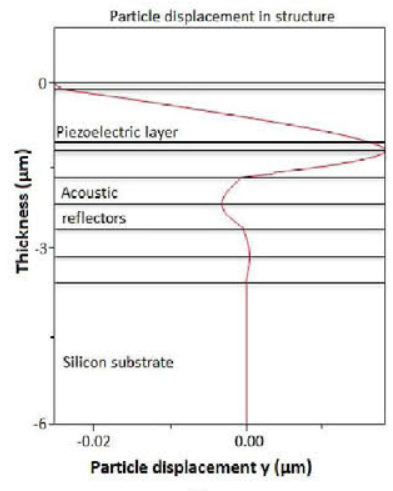

(a)

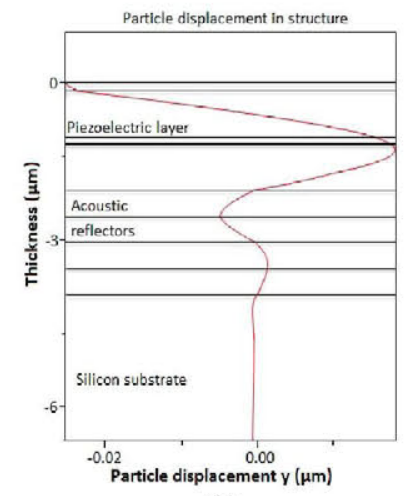

(b)
Fig. 9. A $\lambda / 2$ mode type (a) symmetric and (b) asymmetric solidly mounted resonators.

influence on the TCF of asymmetrizing this structure by increasing or decreasing the thickness of the different layers of the SMRs. The acoustic isolation of the reflector increases when the total number of layers' increases. After some preliminary test, we have observed that the influence in TCF of each layer strongly decreases with its position in the stack; the uppermost layer has a strong influence, which quickly decrease as the layer is far from the piezoelectric layer. This is predictable as the energy entering into each layer decreases as this is located deeper in the structure because the energy reflects in each interface between two different acoustic impedance materials. Fig. 9 shows the amplitude of the standing wave as a function of the layer position. It is calculated with the 2D finite element modeling using perturbation method. First, it exploits the linear acoustics method to evaluate nonlinear second-order force effects. Second, the acoustic equations are solved in the frequency domain. Note the different distribution of the standing part in the asymmetric part of the reflector. It can be observed that the amplitude of the wave becomes smaller as moving away from the piezoelectric film. As a consequence, the influence of the thermal effects occurring in the deeper layers are very low in the reflector performance. Therefore, we have only studied the variations in thicknesses of the uppermost low acoustic impedance material layer, just below the bottom electrode, and of the following high acoustic material layer, the second in order from top to bottom. The rest of the layers, to a total of seven, have always the quarterwavelength thickness. The amplitude becomes nearly zero in the substrate which means that a small acoustic energy leaks to the substrate.

In most of the materials, the elastic constants decrease when increasing temperature. This implies a reduction in the acoustic wave velocity of any propagating mode and, therefore, in the resonant frequency of each mode, which is affected by those materials. This leads to a negative TCF. However, some special materials such as $\mathrm{SiO}_{2}$ [26] and invar alloy [27] have positive temperature coefficient of the elastic constant making the acoustic velocity to increase with temperature. If one of this material is used as component in a SMR, the TCF varies toward positive (usually, less negative) because the shift of the frequency to lower values due to the decreasing of the acoustic velocities is partially compensated by the increase of this magnitude in the mentioned material. As we mentioned earlier, the extent of this compensation strongly depends on the influence of the compensating material on the resonant frequency of the whole device. This influence is related with the amount of acoustic energy fed into the material by the traveling acoustic waves. $\mathrm{SiO}_{2}$ has a special interest because it has several advantages for being a constituent of the acoustic reflector in a SMR. First, its acoustic impedance is low enough because its density is low; secondly, it is a commonly used material in electronic technology and, therefore the deposition methods and properties are very well known; finally, the temperature coefficient of elastic constants is negative below $40 \mathrm{~K}$ and becomes positive above this temperature. Above this temperature, the thermal energy is used for exciting the transverse vibrational modes of Si-O-Si bonding oxygen instead of stretching vibrational mode of the $\mathrm{Si}-\mathrm{O}$ bond. With an increase in temperature, the amplitude of the transverse vibration mode is also increases. This transverse vibrational mode is shortening the average distance between two silicon atoms. This causes to increase in apparent force constant which leads to an increase in elastic modulus and, therefore in sound velocity, which results in the positive temperature coefficient of sound velocities. If there is a further decrease in the average interatomic distance, then it contributes to negative thermal expansion coefficient [14], which also favors the compensation of negative TCF of resonators.

The increase in the thickness of $\mathrm{SiO}_{2}$ also has detrimental effects. It causes the decrease of resonant frequency, and of $k_{\text {eff }}^{2}$ and $Q$. A careful design of the piezoelectric stack (electrode and AlN thicknesses) allows to recover the frequency value and reduces the resonator worsening. The variation of the thickness of any layer of the reflector changes its response. If the thickness is increased beyond the specific value (different for each material), the response of the reflector is badly affected by reducing its reflectivity at the working frequency.

The TCF at resonant frequency is determined by the following relation.

$$
T C F=\frac{1}{f_{0}} \frac{d f}{d T}
$$

Where $f_{0}$ is the resonant or anti-resonant frequency at room temperature. $d f / d T$ is the fitted slope of resonant or antiresonant frequency versus temperature. The temperature coefficient is usually expressed in parts-per-million-per-degree Celsius $\left(\mathrm{ppm} /{ }^{\circ} \mathrm{C}\right)$.

As we mentioned above, the origin of TCF is related to the temperature variations of the elastic constants and the linear expansion coefficient $\alpha$, which affects the mass density, dielectric constant, and the geometry of the resonator (area and thicknesses). However, the most important contribution is due to the variations in the elastic constants and we have only consider the variations of the acoustic velocity with temperature as the responsible of TCF.

To study the influence of the two uppermost layers of the acoustic reflector on the TCF of the resonators we have calculated this coefficient when varying the thickness of those layers. First we will present the evolution of the TCF of 


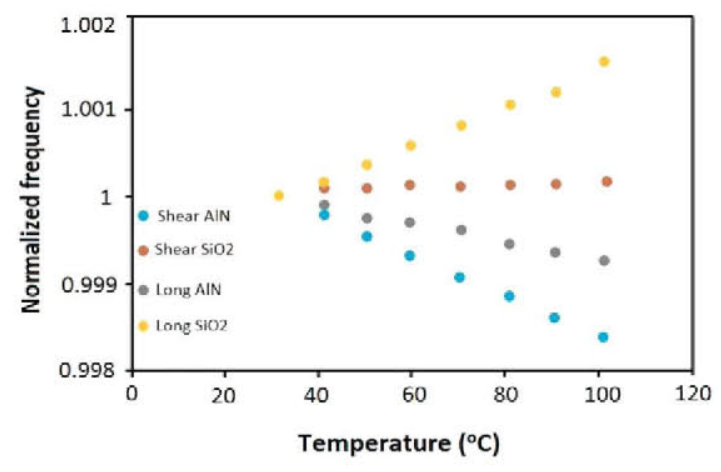

Fig. 10. Measurements of normalized resonant frequency v/s temperature of shear and longitudinal modes for AlN-based resonator with asymmetric Mo-reflector (uppermost $\mathrm{SiO}_{2}$ reflector layer of $1.21 \mu \mathrm{m}$ ).

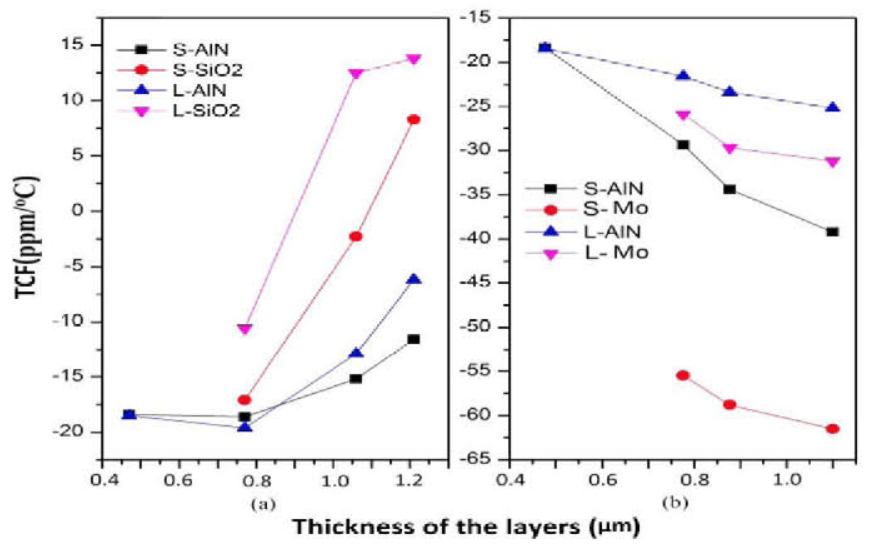

Fig. 11. Measured values of TCF of the main (AlN) and secondary $\left(\mathrm{Mo}\right.$ or $\mathrm{SiO}_{2}$ ) shear $(\mathrm{S})$ and longitudinal $(\mathrm{L})$ modes for asymmetric resonators as a function of the thickness of $\mathrm{SiO}_{2}$ (a) and $\mathrm{Mo}$ (b) uppermost layers in SMR reflectors.

the shear and longitudinal modes with the thickness of the uppermost $\mathrm{SiO}_{2}$ layer of SMRs made with $\mathrm{Mo}$ and $\mathrm{Ta}_{2} \mathrm{O}_{5}$ as high impedance materials and in second place the same evolution but varying the thickness of the uppermost high acoustic impedance material layer for each type of reflector.

The thermal variation of the elastic constant of materials contributes to the TCF through the acoustic velocity. Numerical simulations show that other thermal effects such as thermal expansion are low enough and only contribute up to $2 \%$ of the TCF. Therefore, the only source of TCF we have considered is the thermal variation of sound velocity. Fig.10 shows the normalized resonance frequency as a function of temperature for shear and longitudinal modes as shown in Fig.6. Asymmetric Mo-based reflectors are used in this configuration with the thickness of the uppermost $\mathrm{SiO}_{2}$ reflector layer of $1.21 \mu \mathrm{m}$.

The TCF obtained are $-6.2 \mathrm{ppm} /{ }^{\circ} \mathrm{C}$ and $-11.6 \mathrm{ppm} /{ }^{\circ} \mathrm{C}$ for the longitudinal and shear wave modes of $\mathrm{AlN}$ and $+13.8 \mathrm{ppm} /{ }^{\circ} \mathrm{C}$ and $+8.28 \mathrm{ppm} /{ }^{\circ} \mathrm{C}$ for the longitudinal and shear overtones in $\mathrm{SiO}_{2}$ layers.

The TCF variations of the resonator with main longitudinal mode frequency at $3.3 \mathrm{GHz}$ frequency made of $\mathrm{SiO}_{2}-\mathrm{Mo}$ reflectors is achieved by increasing the thickness of either $\mathrm{SiO}_{2}$ or Mo layers as shown in Fig.11. The result is according to the prediction.
TABLE II

TCF MEASUREMENTS OF SEVERAL RESONATORS With SyMmETRIC AND AS YMMETRIC $\mathrm{SiO}_{2} / \mathrm{Mo}$ REFLECTORS VARYING Mo OR $\mathrm{SiO}_{2}$ UPPERMOST ACOUSTIC REFLECTOR L AYERS

\begin{tabular}{|c|c|c|c|c|}
\hline $\begin{array}{c}\text { Thickness } \\
(\mu \mathrm{m})\end{array}$ & S-AIN & ${\mathrm{S}-\mathrm{SiO}_{2}}_{2}$ & L-AlN & $\mathrm{L}^{-S_{i O}}$ \\
\hline \multicolumn{5}{|l|}{ Symmetric } \\
\hline Reflector & -18.4 & - & -18.5 & - \\
\hline $\begin{array}{c}\text { Asymmetric } \\
\mathrm{Reflector} \\
\mathrm{SiO}_{2}=0.77\end{array}$ & -18.6 & -17.1 & -19.6 & -10.6 \\
\hline $\begin{array}{c}\text { Asymmetric } \\
\text { Reflector } \\
\mathrm{SiO}_{2}=1.06\end{array}$ & -15.2 & -2.3 & -12.9 & +12.5 \\
\hline \multirow{2}{*}{$\begin{array}{c}\text { Asymmetric } \\
\text { Reflector } \\
\mathrm{SiO}_{2}=1.21 \\
\end{array}$} & -11.6 & +8.28 & -6.2 & +13.8 \\
\hline & S-AIN & S-Mo & L-AIN & L-Mo \\
\hline $\begin{array}{c}\text { Asymmetric } \\
\text { Reflector } \\
\text { Mo }=0.77\end{array}$ & -29.4 & -55.5 & -21.6 & -25.9 \\
\hline $\begin{array}{c}\text { Asymmetric } \\
\text { Reflector } \\
\text { Mo }=0.87\end{array}$ & -34.4 & -58.8 & -23.4 & -29.7 \\
\hline $\begin{array}{c}\text { Asymmetric } \\
\text { Reflector } \\
\mathrm{Mo}=1.1\end{array}$ & -39.2 & -61.5 & -25.2 & -31.2 \\
\hline
\end{tabular}

When the thickness of $\mathrm{SiO}_{2}$ is increased to $0.77 \mu \mathrm{m}$, the TCF of L-AlN is increased slightly in negative value. It is possibly due to the coupling of sound velocities of the high and low acoustic impedance materials which results in the negative increase. The data of the TCF for the symmetric and asymmetric resonators using Mo as high impedance material with shear and longitudinal wave mode for varying $\mathrm{Mo}$ or $\mathrm{SiO}_{2}$ uppermost reflector layer are given in the Table II.

The acoustic reflectors are well designed and fabricated for shear and longitudinal mode with different thicknesses of the $\mathrm{SiO}_{2}$ and $\mathrm{Mo}$ uppermost layers of the reflector. The symmetric reflector only shows two resonances corresponding to the main AlN shear and longitudinal modes, similar to the impedance spectrum showed in Fig.6. When the thickness of $\mathrm{SiO}_{2}$ is increased, two additional overtone modes corresponding to shear and longitudinal waves resonating in this layer can be observed. This is because their frequencies become into the reflective bands of the acoustic mirrors. The shear and longitudinal mode of AlN shows a reduction of TCF from their negative value with the increase of $\mathrm{SiO}_{2}$ thickness. As, $\mathrm{SiO}_{2}$ has positive temperature coefficient of sound velocity, an increase in the thickness of $\mathrm{SiO}_{2}$ causes TCF to become less negative and even turning to positive when the thickness of $\mathrm{SiO}_{2}$ is more than 1um. Further increase in the thickness of $\mathrm{SiO}_{2}$ causes the shifts of the resonant and antiresonant frequencies. The best possible compensation of TCF is done by increasing the thickness of $\mathrm{SiO}_{2}$ around $1.21 \mathrm{um}$ as given in table II. The further increase in the thickness of $\mathrm{SiO}_{2}$ can also do the TCF more positive but with the cost of a great energy leakage from reflector. The reflector response is badly affected beyond this thickness. 
TABLE III

TCF $\left(\mathrm{ppm} /{ }^{\circ} \mathrm{C}\right)$ MEASUREMENTS OF SEVERAL REsonators With SYMMETRIC AND ASYMMETRIC $\mathrm{Ta}_{2} \mathrm{O}_{5}$ REFLECTORS

\begin{tabular}{|c|c|c|c|c|}
\hline $\begin{array}{c}\text { Thickness } \\
(\mu \mathrm{m})\end{array}$ & S-AIN & 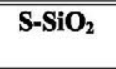 & L-AIN & $\mathrm{L}_{\mathrm{L}-\mathrm{SiO}_{2}}$ \\
\hline \multicolumn{5}{|l|}{ Symmetric } \\
\hline Reflector & -30.9 & - & -17.8 & - \\
\hline \multicolumn{5}{|l|}{ Asymmetric } \\
\hline $\begin{array}{l}\text { Reflector } \\
\mathrm{SiO}_{2}=0.82\end{array}$ & -23.2 & -32.6 & -10.5 & -15.4 \\
\hline \multicolumn{5}{|l|}{ Asymmetric } \\
\hline $\begin{array}{l}\text { Reflector } \\
\mathrm{SiO}_{2}=0.98\end{array}$ & -20.7 & -25.9 & -10.7 & +2.00 \\
\hline \multirow{2}{*}{$\begin{array}{c}\text { Asymmetric } \\
\text {-Reflector } \\
\mathrm{SiO}_{2}=1.3\end{array}$} & -17.6 & +7.8 & -6.5 & +12.8 \\
\hline & S-AIN & S-Ta ${ }_{2} \mathrm{O}_{5}$ & L-AIN & L-Ta $\mathbf{T a}_{2} \mathrm{O}_{5}$ \\
\hline $\begin{array}{l}\text { Asymmetric } \\
\text { Reflector } \\
\mathrm{Ta}_{2} \mathrm{O}_{5}=0.71\end{array}$ & -27.5 & -28.5 & -17.8 & -23.9 \\
\hline \multicolumn{5}{|l|}{ Asymmetric } \\
\hline $\begin{array}{l}\text { Reflector } \\
\mathrm{Ta}_{2} \mathrm{O}_{5}=0.84\end{array}$ & -27.7 & -29.2 & -29.7 & -22.5 \\
\hline $\begin{array}{l}\text { Asymmetric } \\
\text { Reflector } \\
\mathrm{Ta}_{2} \mathrm{O}_{5}=1\end{array}$ & -38.3 & -34.9 & -31.1 & -35.3 \\
\hline
\end{tabular}

Likewise, the thickness of Mo is also varying to observe its effect on TCF. The increase in the thickness of Mo causes a rapid increase in the TCF of shear mode for Mo in negative values than the shear and longitudinal mode of AlN. Further increase in the thickness of Mo causes the negative increase in TCF. This shows the influence of Mo as first high impedance layer on the TCF of the resonator. Further increase in Mo thickness causes distortion of acoustic reflector response towards energy leakage. The reduction in the thickness of Mo can make TCF more negative because with the increase in temperature the sound velocity decreases in Mo.

The TCF of $\mathrm{Ta}_{2} \mathrm{O}_{5}$ based symmetric and asymmetric resonators in shear and a longitudinal wave mode are also investigated. The results are given in Table III.

The resonators based on simulation of $\mathrm{Ta}_{2} \mathrm{O}_{5}$-reflectors exhibits an anomalous behavior as shown in Fig.12(a).

The increase in the thickness of $\mathrm{Ta}_{2} \mathrm{O}_{5}$ should lead to a decrease (more negative) of the TCF because of its negative temperature coefficient, but it shows an increase in simulated values as shown in Fig. 12(a). This kind of behavior is also observed in experimental results for longitudinal wave mode in some range of increasing thickness of $\mathrm{Ta}_{2} \mathrm{O}_{5}, \mathrm{Fig} .12(\mathrm{~b})$. When the thickness of $\mathrm{Ta}_{2} \mathrm{O}_{5}$ is increased from $0.64 \mu \mathrm{m}$ to $0.84 \mu \mathrm{m}$, then there is a positive increase in TCF values of $\mathrm{Ta}_{2} \mathrm{O}_{5}$ which satisfy our simulation result for this range. Further increase in $\mathrm{Ta}_{2} \mathrm{O}_{5}$ thickness leads to the negative increase of TCF. To find the reason behind this behavior, we have looked at the structure of $\mathrm{Ta}_{2} \mathrm{O}_{5}$. There are two types of oxygen vacancy defects present in $\mathrm{Ta}_{2} \mathrm{O}_{5}$ structure [28]. One is in-plane type vacancy that results in a shallow unoccupied level. The other is

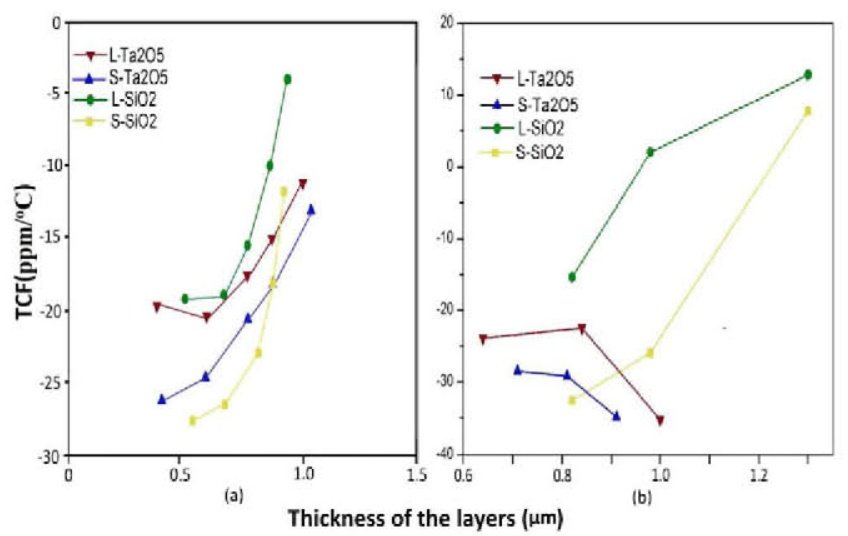

Fig. 12. Simulated (a) and measured (b) values of TCF as a function of the $\mathrm{Ta}_{2} \mathrm{O}_{5}$ layer thickness in asymmetric reflectors.

cap-type vacancy presents in the occupied and unoccupied states of the conduction band. Most probably, this kind of behavior is due to the presence of these vacancies. As the thermal variation in longitudinal elastic constants $\left(\mathrm{c}_{33}\right.$ and $\left.\mathrm{c}_{44}\right)$ provides the main contribution to the TCF because they set the acoustic velocity with the mass density $\left(v_{1}=\left(c_{\mathrm{xx}} / \rho\right)^{1 / 2}\right)$. The material expansion coefficients are too low to have a first order effect on the resonant frequency by varying the thickness and mass density. Although this effect is very small but on the individual material level it cannot be neglected. Second possible reason is the sound velocity coupling of the low and high acoustic impedance materials because the sound velocity of $\mathrm{SiO}_{2}$ is lower than that of Mo but it is considerably higher than that of $\mathrm{Ta}_{2} \mathrm{O}_{5}$.

The experimentally calculated values of TCF of $\mathrm{Ta}_{2} \mathrm{O}_{5}$ based resonators are given in the Table III. A positive increase in the values of TCF for both shear and longitudinal can be seen by increasing the thickness of $\mathrm{SiO}_{2}$ layer just below the piezoelectric stack. By increasing the value up to $1.3 \mu \mathrm{m}$, the longitudinal and shear wave mode of TCF shows a positive increase and their values becomes $-6.5\left(\mathrm{ppm} /{ }^{\circ} \mathrm{C}\right)$ and $-17.6\left(\mathrm{ppm} /{ }^{\circ} \mathrm{C}\right)$ for $\mathrm{AlN}$ and $+12.8\left(\mathrm{ppm} /{ }^{\circ} \mathrm{C}\right)$ and $+7.8\left(\mathrm{ppm} /{ }^{\circ} \mathrm{C}\right)$ for $\mathrm{SiO}_{2}$ respectively. By decreasing the thickness of $\mathrm{Ta}_{2} \mathrm{O}_{5}$ layer, the TCF of both longitudinal and shear resonance frequencies become less negative as given in Table III. At a higher frequency, the thickness of high impedance layers can only increase up to a certain limit, but at a lower frequency, this limit is exceeded.

The change in resonant frequency and quality factor with the rise in temperature is a matter of interest when SMRs are applied in any application. Fig. 13 shows these changes as a function of temperature for one of the symmetric and asymmetric (at maximum compensation of TCF) SMRs.

In Fig.13(a), with the increase in temperature there is a slight decrease in the resonant frequency but the quality factor increases in sufficient amount simultaneously. When the thickness of first acoustic reflector layer (i.e. $\mathrm{SiO}_{2}$ ) increases to compensate TCF, the quality factor is even more better than symmetric configurations. There is a decrease in resonant frequency as compared to symmetric SMR and it is due to the mass loading effect which is caused by the heavy layer 

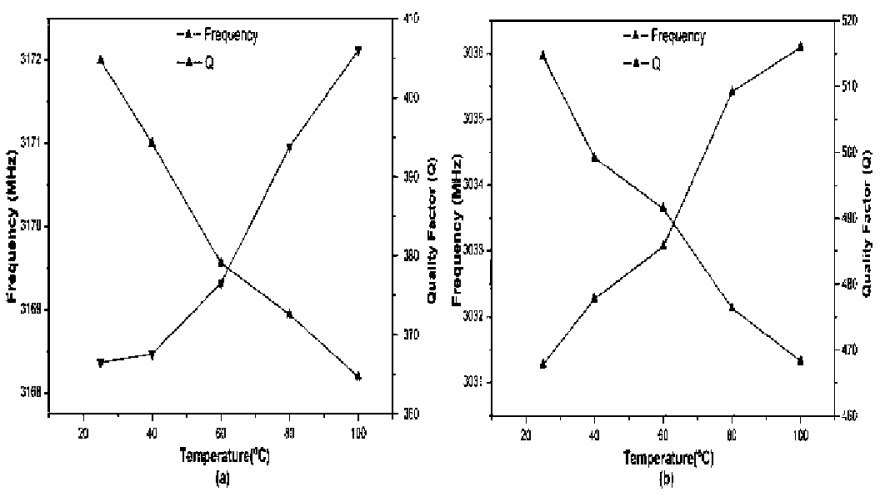

Fig. 13. Resonant frequency and quality factor as a function of temperature for (a) symmetric and (b) asymmetric SMRs.

of $\mathrm{SiO}_{2}$. Fig.13 shows that the quality factor is improved with our design and best compensation of TCF is also achieved.

In summary, it is possible to completely compensate the TCF of longitudinal wave mode at high frequency by adjusting the thickness of top low and high impedance layers below piezoelectric stack. The introduction of new insulating materials such as $\mathrm{Ta}_{2} \mathrm{O}_{5}$ can further improve TCF. By increasing the number of layers, reflection loss of the reflectors can be improved but it has negligible effect on the TCF of the resonators.

\section{CONCLUSION}

The influence of the different material with specified thickness on the TCF of the symmetric and asymmetric AlN based SMRs is studied in detail. The TCF for shear and longitudinal modes for each thickness of high and low impedance layers are calculated. The TCF for longitudinal modes can be compensate to $0 \mathrm{ppm} /{ }^{\circ} \mathrm{C}$ without much degrading the performance of SMRs. For simulation purpose Mason model and Finite Element Analysis are used. Experimental results were obtained by fabricating asymmetric reflectors by varying the thickness of one of the uppermost reflector layers (low impedance $\mathrm{SiO}_{2}$ or high impedance one, Mo or $\mathrm{Ta}_{2} \mathrm{O}_{5}$ ). It is observed from both simulation and experimental results that TCF is mainly depends on the thickness of top two layers just below the piezoelectric stack. An increase of the $\mathrm{SiO}_{2}$ layer to near two times the ideal $\lambda / 4$-thickness strongly compensate the AlN resonant frequencies of both longitudinal and shear modes, making the $\mathrm{SiO}_{2}$ generated overtones to have a positive TCF. The variation of the uppermost high acoustic impedance layer thickness ( $\mathrm{Mo}$ or $\mathrm{Ta}_{2} \mathrm{O}_{5}$ ) do not have the same result making the TCF of all modes to be more negative than in the symmetric reflector. However, the simulations show a contrary behavior for $\mathrm{Ta}_{2} \mathrm{O}_{5}$. Although this is not clearly explained, one should think that the possibility of some kind of interaction of the particular layer properties than has not been obtained experimentally.

\section{REFERENCES}

[1] U. Bauernschmitt, C. Block, P. Hagn, G. Kovacs, A. Przadka, and C. C. W. Ruppel, "Concepts for RF front-ends for multi-mode, multiband cellular phones," in Proc. Eur. Conf. Wireless Technol., 2007, pp. $130-133$.
[2] J. Weber et al., "Shear mode FBARs as highly sensitive liquid biosensors," Sens. Actuators A, Phys., vol. 128, no. 1, pp. 84-88, 2006.

[3] G. Wingqvist, J. Bjurstrom, L. Liljeholm, I. Katardjiev, and A. L. Spetz, "Shear mode AlN thin film electroacoustic resonator for biosensor applications," in Proc. IEEE SENSORS, Mar. 2005, p. 4.

[4] I. Katardjiev and V. Yantchev, "Recent developments in thin film electroacoustic technology for biosensor applications," Vacuum, vol. 86, no. 5, pp. 520-531, 2012.

[5] D. Chen et al., "Micro-electromechanical film bulk acoustic sensor for plasma and whole blood coagulation monitoring," Biosens. Bioelectron., vol. 91, pp. 465-471, May 2017.

[6] D.Chen J. Wang, P. Wang, Q. Guo, Z. Zhang, and J. Ma, "Real-time monitoring of human blood clotting using a lateral excited film bulk acoustic resonator," J. Micromech. Microeng., vol. 27, no. 4, p. 045013, 2017.

[7] K. M. Lakin, K. T. McCarron, J. F. McDonald, and J. Belsick, "Temperature coefficient and ageing of BAW composite materials," in Proc. IEEE Int. Freq. Control Symp. PDA Exhibit., Jun. 2001, pp. 605-608.

[8] W. Heeren, M. Fattinger, G. Fattinger, A. Volatier, and R. Aigner, "Impact of thermo-mechanical stress on the TCF of WLP BAW filters," in Proc. IEEE Int. Ultrason. Symp. (IUS), Sep. 2016, pp. 1-4.

[9] A. Volatier, G. Fattinger, F. Dumont, P. Stoyanov, and R. Aigner, "Technology enhancements for high performance BAW duplexer," in Proc. IEEE Int. Ultrason. Symp. (IUS), Jul. 2013, pp. 761-764.

[10] D. Ekeom and B. Dubus, "Thermoelastic FEM-BEM model for solidly mounted resonator," in Proc. IEEE Ultrason. Symp. (IUS), Nov. 2008, pp. $1564-1567$.

[11] D. Chen, W. Ren, S. Song, J. Wang, W. Liu, and P. Wang, "The high Q factor lateral field-excited thickness shear mode film bulk acoustic resonator working in liquid," Micromachines, vol. 7, no. 12, p. 231, 2016.

[12] K. Onishi et al., "A novel temperature compensation method for SAW devices using direct bonding techniques," in Proc. IEEE Ultrason. Symp., Oct. 1997, pp. 227-230.

[13] H. Zhang, Q. Yang, W. Pang, J. G. Ma, and H. Yu, "Temperature stable bulk acoustic wave filters enabling integration of a mobile television function in UMTS system," IEEE Microw. Wireless Compon. Lett., vol. 22, no. 5, pp. 239-241, May 2012.

[14] B. Ivira, P. Benech, R. Fillit, F. Ndagijimana, P. Ancey, and G. Parat, "Modeling for temperature compensation and temperature characterizations of BAW resonators at GHz frequencies," IEEE Trans. Ultrason. Ferroelect., Freq. Control, vol. 55, no. 2, pp. 421-430, Feb. 2008.

[15] J.-S. Shin et al., "Hybrid bulk acoustic wave structure for temperature stability in LTE applications," IEEE Microw. Wireless Compon. Lett., vol. 23, no. 9, pp. 453-455, Sep. 2013.

[16] T. Nishihara, S. Taniguchi, and M. Ueda, "Increased piezoelectric coupling factor in temperature-compensated film bulk acoustic resonators," in Proc. IEEE Int. Ultrason. Symp. (IUS), Oct. 2015, pp. $1-4$.

[17] W. P. Mason and H. Baerwald, "Piezoelectric crystals and their applications to ultrasonics," Phys. Today, vol. 4, no. 5, p. 23, 1951.

[18] S. Marksteiner, J. Kaitila, G. G. Fattinger, and R. Aigner, "Optimization of acoustic mirrors for solidly mounted BAW resonators," in Proc. IEEE Int. Ultrason. Symp., Sep. 2005, pp. 329-332.

[19] K. M. Lakin, K. T. McCarron, and R. E. Rose, "Solidly mounted resonators and filters," in Proc. IEEE Int. Ultrason. Symp., Nov. 1995, pp. 905-908.

[20] M. DeMiguel-Ramos, T. Mirea, J. Olivares, M. Clement, J. Sangrador, and E. Iborra, "Assessment of the shear acoustic velocities in the different materials composing a high frequency solidly mounted resonator," Ultrasonics, vol. 62, pp. 195-199, Sep. 2015.

[21] J. Olivares et al., "Sputtered $\mathrm{SiO}_{2}$ as low acoustic impedance material for Bragg mirror fabrication in BAW resonators," IEEE Trans. Ultrason., Ferroelect., Freq. Control, vol. 57, no. 1, pp. 23-29, Jan. 2010.

[22] J. Capilla, J. Olivares, M. Clement, J. Sangrador, E. Iborra, and A. Devos, "High-acoustic-impedance tantalum oxide layers for insulating acoustic reflectors," IEEE Trans. Ultrason., Ferroelect., Freq. Control, vol. 59, no. 3, pp. 366-372, Mar. 2012.

[23] J. Kaitila, "3C-1 review of wave propagation in BAW thin film devicesProgress and prospects," in Proc. IEEE Ultrason. Symp., Oct. 2007, pp. $120-129$.

[24] M. DeMiguel-Ramos, T. Mirea, M. Clement, J. Olivares, J. Sangrador, and E. Iborra, "Optimized tilted c-axis AlN films for improved operation of shear mode resonators," Thin Solid Films, vol. 590, pp. 219-223, Sep. 2015. 
[25] H. Kanbara, H. Kobayashi, and K. Nakamura, "Analysis of piezoelectric thin film resonators with acoustic quarter-wave multilayers," Jpn. J. Appl. Phys., vol. 39, no. 5S, p. 3049, 2000.

[26] S. M. Matsuda et al., "Correlation between temperature coefficient of elasticity and Fourier transform infrared spectra of silicon dioxide films for surface acoustic wave devices," IEEE Trans. Ultrason., Ferroelect., Freq. Control, vol. 58, no. 8, pp. 1684-1687, Aug. 2011.

[27] L. Maosa, G. A. Saunders, H. Rahdi, U. Kawald, J. Pelzl, and H. Bach, "Acoustic-mode vibrational anharmonicity related to the anomalous thermal expansion of Invar iron alloys," Phys. Rev. B, Condens. Matter, vol. 45 , no. 5 , p. $2224,1992$.

[28] R. Ramprasad, "First principles study of oxygen vacancy defects in tantalum pentoxide," J. Appl. Phys., vol. 94, no. 9, pp. 5609-5612, 2003. 